Disponible en ligne :

www.cahiersagricultures.fr

\title{
Incidence de l'ombrage sur les caractères phénotypiques du cacaoyer en zone de transition forêt-savane au centre de la Côte d'Ivoire
}

\author{
Venance-Pâques Gniayou Kouadio, ${ }^{1,}$, Bi Tra Aimé Vroh ${ }^{1}$, Kouassi Bruno Kpangui ${ }^{2}$, \\ Affia Sonmia Francia Kossonou ${ }^{1}$ et Constant Yves Adou Yao ${ }^{1,3}$ \\ ${ }^{1}$ Équipe de recherche BioValSE, Laboratoire de botanique, UFR Biosciences, Université Félix Houphouët-Boigny, Abidjan, Côte d'Ivoire \\ ${ }^{2}$ UFR Environnement, Université Jean Lorougnon Guédé, Daloa, Côte d'Ivoire \\ ${ }^{3}$ Centre suisse de recherches scientifiques en Côte d'Ivoire (CSRS), Abidjan, Côte d'Ivoire
}

\begin{abstract}
Résumé - Le centre de la Côte d'Ivoire abrite divers systèmes agroforestiers à base de cacaoyers, qui se distinguent selon la densité de l'ombrage des arbres associés : système simple (canopée ouverte et espèces exotiques - bananier et avocatier), mixte (canopée ouverte, peu d'espèces locales) ou complexe (canopée fermée et nombreuses espèces locales). La floraison et la fructification de 220 cacaoyers ont été suivies dans 44 parcelles de $625 \mathrm{~m}^{2}$, réparties dans les trois systèmes. Le nombre de coussinets floraux, le nombre de cabosses mûres par cacaoyer, ainsi que la masse et la qualité des cabosses sont significativement plus grands dans le système simple que dans le système complexe. Bien que le nombre moyen de fèves par cabosse ne varie pas significativement, la masse moyenne des fèves est plus grande dans le système simple que dans le système complexe. Le système simple serait donc plus productif; cependant, la prise en compte de la valeur des services rendus par les espèces associées devra être prise en compte pour préciser la durabilité.
\end{abstract}

Mots clés : systèmes agroforestiers / cacaoculture durable / contact forêt-savane / services écosystémiques / Côte d'Ivoire

\begin{abstract}
Effects of shade on phenotypic characters of cocoa tree in the forest-savannah transition zone in central Côte d'Ivoire. Central Côte d'Ivoire houses various cocoa-based agroforestry systems, which are distinguished by the density of shade trees: simple (open canopy, exotic species - banana and avocado), mixed (open canopy, few local species), and complex (closed canopy, numerous local species) systems. Flowering and fructification of 220 cocoa trees were monitored in 44 square plots of $625 \mathrm{~m}^{2}$, distributed in the three systems. The number of flowering pads, the number of ripe pods per cocoa tree and the mass and quality of cocoa pods are significantly higher in simple systems than in complex ones. Although the number of beans is not significantly different, the mass of beans is bigger in simple systems than in complex ones. The simple system appears to be the most productive; however, determining the value of the services rendered by the associated species will be necessary to assess sustainability.
\end{abstract}

Keywords: agroforestry system / sustainable cocoa farming / forest-savannah contact / ecosystem services / Côte d'Ivoire

\section{Introduction}

La zone de contact forêt-savane au centre de la Côte d'Ivoire, autrefois constituée d'une mosaïque de savanes guinéennes et de forêts denses humides semi-décidues (Guillaumet et Adjanohoun, 1971), est aujourd'hui dominée

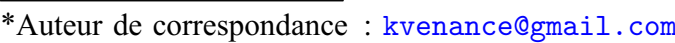

par la mosaïque culture-jachère (Kpangui, 2015). Les cultures de rente (café et cacao) ont été introduites entre 1928 et 1930 au détriment des surfaces forestières (Chauveau, 1979). Aujourd'hui, si les plantations de caféiers ont pratiquement disparu de la zone, celles de cacaoyers se sont développées et se pratiquent même dans les zones de savanes, alors qu'elles étaient autrefois jugées inaptes à la pratique cacaoyère (Herzog, 1994; Camara et al., 2009): longue saison sèche, pluviométrie annuelle moyenne de $1090 \mathrm{~mm}$ et sols de faible 


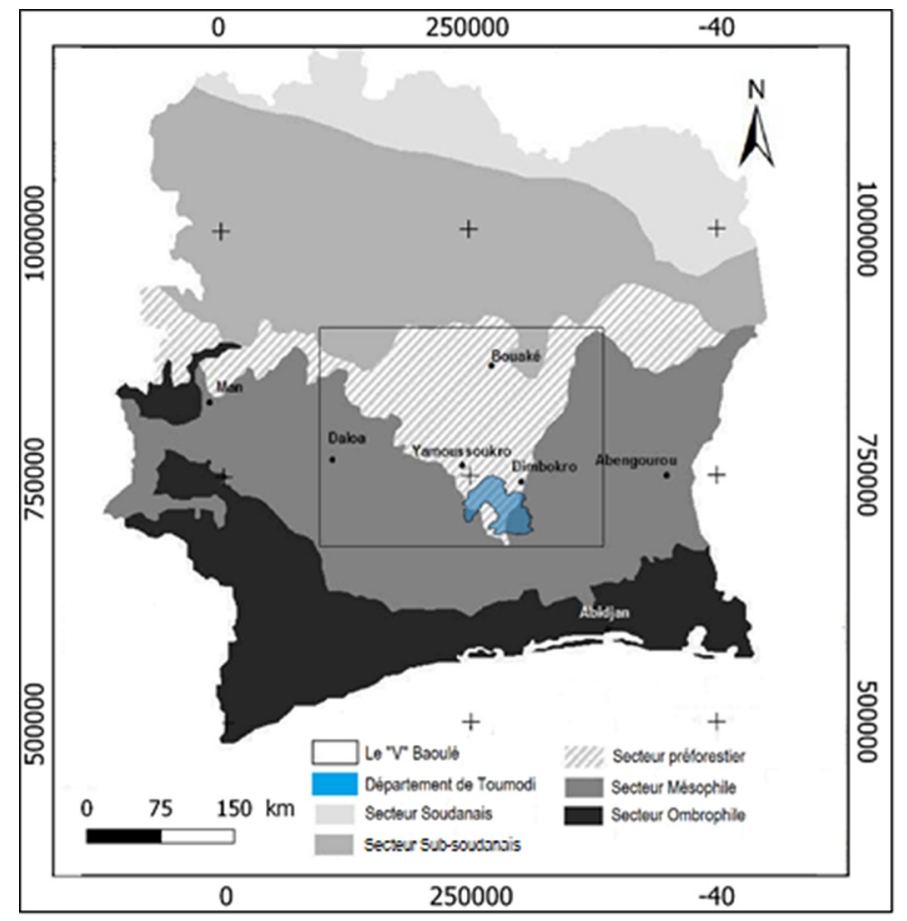

Fig. 1. Localisation de la mosaïque forêt-savane dans la région du «V Baoulé». Source: Hiernaux, 1975, modifié par Kpangui, 2014.

Fig. 1. Localization of the forest-savannah transition zone in the "V Baoule" region.

fertilité; les déficits hydriques des mois secs sont cependant compensés par le pouvoir de rétention en eau des roches vertes et des sols schisteux (Avenard et al., 1973).

Le cacaoyer (Theobroma cacao L.) est cultivé en zone forestière et exige une pluviométrie supérieure à $1100 \mathrm{~mm}$ (Jagoret, 2011), avec une saison sèche atténuée, mais nécessaire à la formation des cabosses. Il exige également une température moyenne annuelle comprise entre $18-21^{\circ} \mathrm{C}$ et $30-32^{\circ} \mathrm{C}$. Sa grande plasticité fait qu'il peut s'adapter à une grande variété de milieux biophysiques, de types de sols et de conditions de luminosité et de pluviométrie (Freud et al., 2000 ; Deheuvels, 2011 ; Jagoret, 2011).

En Côte d'Ivoire, la culture du cacaoyer se pratique principalement dans la moitié sud forestière du pays (Assiri, 2010). Introduite vers 1888 (Bardin, 1937 ; Burle, 1961), elle y a connu une expansion rapide au détriment des surfaces forestières. La cacaoculture extensive et itinérante longtemps pratiquée (Assiri et al., 2009) a engendré la dégradation de l'environnement forestier et de sa biodiversité.

La zone de contact forêt-savane a été longtemps considérée défavorable à la culture du cacaoyer: rareté des terres forestières, fréquence des feux de brousse, pluviosité inférieure aux exigences du cacaoyer et inégalement répartie dans l'année (Blanc-Pamard, 1978; Jagoret et al., 2008). Pourtant, certaines régions de cette zone, telles que l'Est de Dimbokro et l'Ouest de Toumodi, au centre de la Côte d'Ivoire, ont été de grandes zones de production cacaoyère (Fig. 1), où sa réussite est attribuée à la présence de divers systèmes agroforestiers, dans lesquels des espèces locales et exotiques sont associées aux cacaoyers (Herzog, 1994). Alors que selon Sanial (2014), «les systèmes agroforestiers à cacaoyers semblent avoir presque totalement disparu du paysage ivoirien », plusieurs travaux ont mis en évidence la présence de ces systèmes au centre-ouest de la Côte d'Ivoire (Piba, 2008; Cissé, 2013). Plus récemment, les travaux réalisés par Kpangui et al. (2015) ont montré que les systèmes agroforestiers à base de cacaoyers $(\mathrm{SAFc})$ persistent dans la sous-préfecture préforestière de Kokumbo. Ces auteurs ont classé les plantations cacaoyères en trois systèmes, en se basant sur la diversité floristique et la structure de la végétation: les SAFc simples (canopée ouverte et forte proportion d'espèces exotiques : bananier Musa paradisiaca et avocatier Persea americana), les SAFc mixtes (canopée ouverte et faible densité d'espèces locales) et les SAFc complexes (canopée fermée et forte densité d'espèces locales). Comme il n'existe pas d'étude sur le comportement du cacaoyer dans ces différents systèmes, on ne sait pas si la productivité du cacaoyer est différente selon les systèmes considérés, ni quels sont les paramètres agronomiques du cacaoyer qui sont sensibles à ces systèmes. La présente étude vise donc à préciser l'influence des différents SAFc sur les facteurs phénotypiques (floraison, fructification) et la productivité des cacaoyers.

\section{Matériel et méthodes}

\subsection{Zone d'étude}

Les travaux se sont déroulés dans la sous-préfecture de Kokumbo (localités de Niamkey-Konankro et de Langossou), située dans le centre de la Côte d'Ivoire, sur la branche ouest du «V Baoulé » en zone de contact forêt-savane (Fig. 2). 


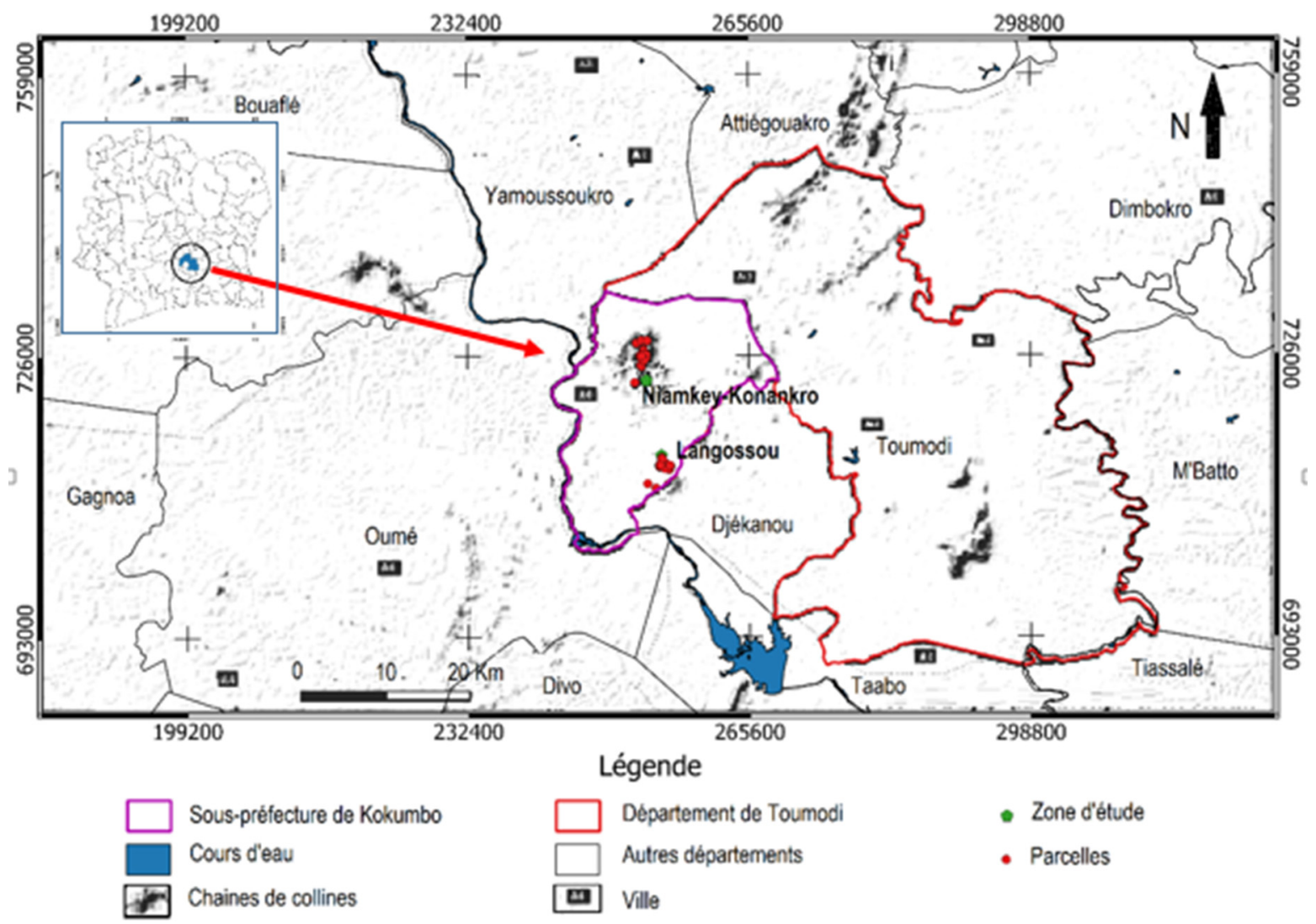

Fig. 2. Localisation de la sous-préfecture de Kokumbo, dans le département de Toumodi, au centre de la Côte d'Ivoire.

Fig. 2. Localization of Kokumbo sous-préfecture, in Toumodi department, in central Côte d'Ivoire.

Le relief du «V Baoulé» est constitué de bas-plateaux et d'un système peu élevé de collines, appelé la « chaîne baoulé ». Les altitudes varient entre $100 \mathrm{~m}$ et $550 \mathrm{~m}$.

Les sols reposent sur de vastes massifs granitiques, et des roches métamorphiques et schisteuses. On distingue les sols ferralitiques et les sols ferrugineux (Perraud, 1971; Peltre, 1977). Les sols ferralitiques, fortement ou moyennement désaturés, sont souvent recouverts d'une forêt sur les sommets des collines et d'une savane sur les pentes. Les sols ferrugineux sont constitués soit d'éléments remaniés assez sableux d'origine ferralitique, soit d'éléments lessivés qui proviennent d'une arène granitique peu profonde (Avenard et al., 1973).

Les précipitations sont caractérisées par deux pics: le premier en juin et le second en octobre. La valeur moyenne des températures est de $27^{\circ} \mathrm{C}$ (Fig. 3).

\subsection{Systèmes agroforestiers à base de cacao}

Dans la zone d'étude, trois systèmes agroforestiers à base de cacao ont été identifiés: simple, mixte et complexe (Kpangui et al., 2015) (Tab.1). Leur gestion par les paysans se fait par le maintien, la préservation ou l'introduction de diverses espèces locales ou exotiques (Kpangui et al., 2015; Adou Yao et al., 2016). La densité de tiges de cacaoyers varie d'un système à un autre: 1076 cacaoyers/ha dans les SAFc simples, 937 cacaoyers/ha dans les SAFc mixtes et 984 cacaoyers/ha dans les SAFc complexes.
Les SAFc complexes se distinguent par une forte présence d'arbres locaux (mégaphanérophytes et mésophanérophytes), perçus par les paysans comme pouvant favoriser la production cacaoyère ou ayant diverses utilisations. La strate dominant les cacaoyers est dense et présente une canopée fermée. Dans ces systèmes complexes est utilisée la variété Amelonado, nommée «cacao français » (Kpangui et al., 2015), la première introduite en Côte d'Ivoire (Ruf et Schroth, 2004). Cette variété nécessite le maintien de l'ombrage pendant toute la durée de la plantation.

Les SAFc simples sont caractérisés par une forte proportion d'espèces exotiques, notamment de bananiers, et une faible proportion d'espèces locales, en plus d'une canopée plus ouverte (Kpangui et al., 2015).

Les SAFc mixtes se situent entre les deux et sont caractérisés par une faible densité d'espèces locales, une forte densité d'espèces exotiques et une canopée ouverte. Les espèces locales sont des arbres mésophanérophytes et microphanérophytes situés dans les première et deuxième strates au-dessus des cacaoyers (Tab. 2).

Ces deux derniers types de SAFc utilisent la variété « cacao Ghana », ou Forastero (haut-amazonienne) ou ses hybrides ( Ruf et Schroth, 2004), qui tolèrent un ombrage modéré ou absent.

\subsection{Collecte des données}

Quarante-quatre parcelles de $625 \mathrm{~m}^{2}(25 \mathrm{~m} \times 25 \mathrm{~m})$ chacune ont été installées, dont 16 dans les SAFc complexes, 11 




Fig. 3. Diagramme ombrothermique du département de Toumodi de 1982 à 2012.

Source : http://www.climate-data.org.

Fig. 3. Ombrothermal diagram of Toumodi department from 1982 to 2012.

Tableau 1. Caractéristique des variables qualitatives et quantitatives des trois SAFc (Kpangui, 2015).

Table 1. Characteristics of qualitative and quantitative variables of the three AFSc.

\begin{tabular}{|c|c|c|c|c|}
\hline & Variables & SAFc simples & SAFc mixtes & SAFc complexes \\
\hline \multirow{2}{*}{ Diversité floristique } & Indice de Shannon & $0,89^{\mathrm{a}}$ & $1,42^{\mathrm{c}}$ & $1,23^{\mathrm{b}}$ \\
\hline & Indice de Simpson & $0,48^{\mathrm{a}}$ & $0,65^{\mathrm{b}}$ & $0,63^{\mathrm{b}}$ \\
\hline \multirow{4}{*}{ Types biologiques } & Géophyte (\%) & $57,9^{\mathrm{c}}$ & $43,5^{b}$ & $9,5^{\mathrm{a}}$ \\
\hline & Microphanérophyte (\%) & $23,0^{\mathrm{a}}$ & $21,7^{\mathrm{a}}$ & $17,7^{\mathrm{a}}$ \\
\hline & Mésophanérophyte (\%) & $15,8^{\mathrm{a}}$ & $32,1^{b}$ & $56,7^{\mathrm{c}}$ \\
\hline & Mégaphanérophyte (\%) & $3,4^{\mathrm{a}}$ & $1,9^{\mathrm{a}}$ & $16,1^{\mathrm{b}}$ \\
\hline Affinités chorologiques & Introduites $(\%)$ & $73,6^{\mathrm{c}}$ & $54,4^{b}$ & $19,5^{\mathrm{a}}$ \\
\hline \multirow[b]{2}{*}{ Structure horizontale des espèces associées } & Densité (tiges/ha) & $352,4^{\mathrm{c}}$ & $210,3^{b}$ & $119,0^{\mathrm{a}}$ \\
\hline & Aire basale $\left(\mathrm{m}^{2} / \mathrm{ha}\right)$ & $13,5^{\mathrm{a}}$ & $18,2^{b}$ & $38,6^{\mathrm{c}}$ \\
\hline \multicolumn{5}{|l|}{ Type d'espèces associées } \\
\hline \multirow[t]{2}{*}{ Forestières } & AGB & $51,2^{\mathrm{a}}$ & $114,3^{b}$ & $239,6^{\mathrm{c}}$ \\
\hline & Densité (tiges/ha) & $32,7^{\mathrm{a}}$ & $80^{\mathrm{b}}$ & $62,8^{\mathrm{b}}$ \\
\hline \multirow[t]{2}{*}{ Exotiques } & AGB & $8,5^{\mathrm{a}}$ & $7,4^{b}$ & $18^{\mathrm{c}}$ \\
\hline & Densité (tiges/ha) & $34,8^{\mathrm{a}}$ & $46,8^{\mathrm{a}}$ & $42,3^{\mathrm{a}}$ \\
\hline Bananiers & AGB & $1,5^{\mathrm{b}}$ & $1,7^{\mathrm{b}}$ & $0,2^{\mathrm{a}}$ \\
\hline
\end{tabular}


Tableau 1. (suite).

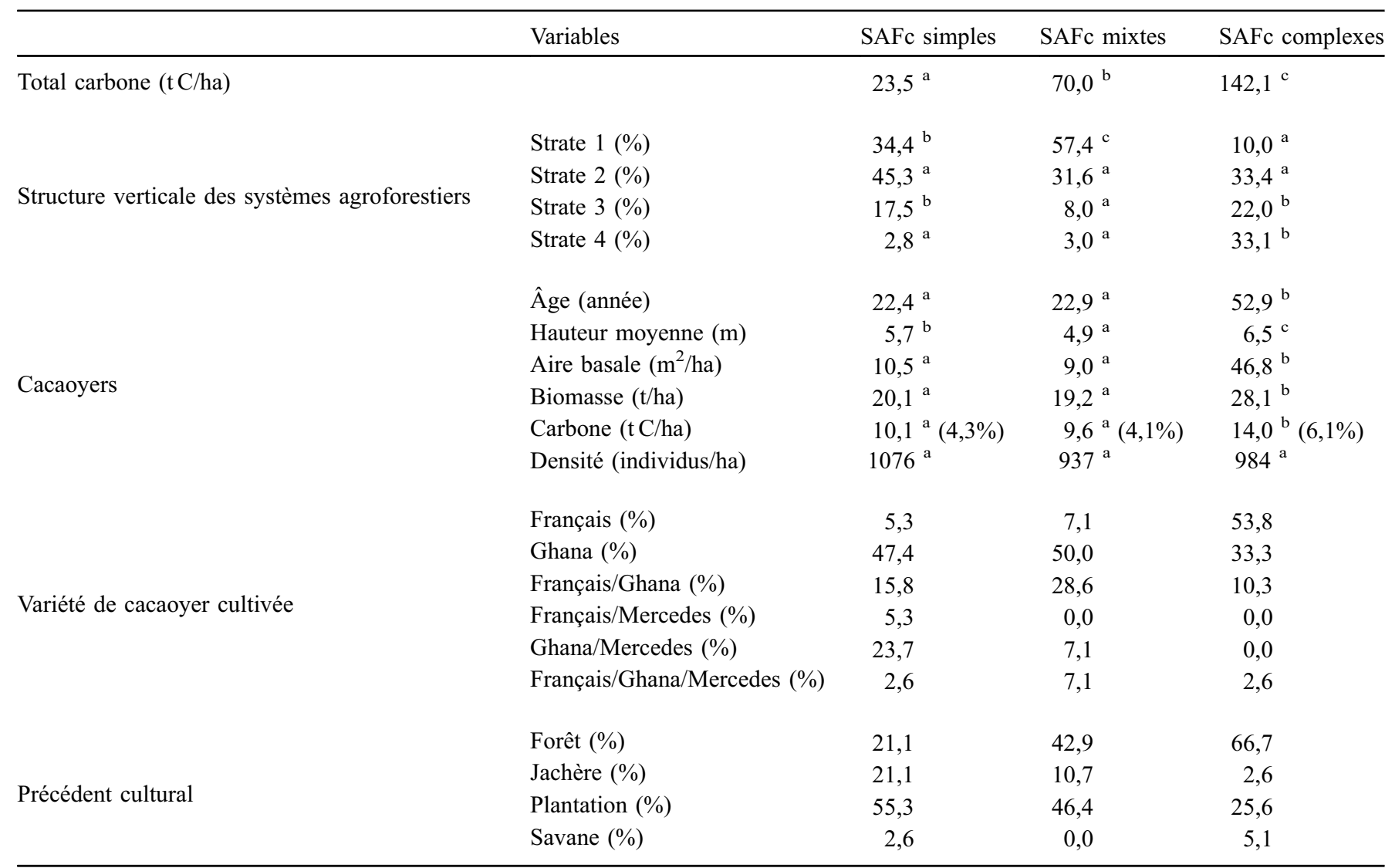

Strate 1 : basse $(2$ à $4 \mathrm{~m})$; Strate $2:$ moyenne $(4$ à $8 \mathrm{~m})$; Strate $3:$ supérieure $(8$ à $16 \mathrm{~m})$; Strate $4:$ émergente (plus de $16 \mathrm{~m}) ; \mathrm{GC}:$ espèce forestière; GC_f: espèce forestière fruitière; GC-SZ: espèce de zone de transition; GC-SZ_f: espèce fruitière de zone de transition; AGB : biomasse aérienne; TC : total de carbone. Mercedes: nouvelle variété hybride peu adoptée dans la zone d'étude.

Pour chaque ligne, les valeurs suivies par une même lettre ne sont pas significativement différentes.

dans les SAFc mixtes et 17 dans les SAFc simples. Dans chacune, 5 cacaoyers ont été sélectionnés pour suivre leur état phénologique de la floraison à la fructification, aux angles et au centre des parcelles. Nous avons distingué les cacaoyers situés sous le houppier ou à proximité d'espèces associées, de ceux qui ne l'étaient pas, de façon à apprécier l'effet de l'ombrage.

\subsection{Paramètres phénotypiques mesurés}

La phénologie des cacaoyers a été suivie toutes les deux semaines de la floraison à la fructification (juin à décembre). Les coussinets floraux formés à une hauteur inférieure à $2 \mathrm{~m}$ et le nombre de chérelles (jeunes fruits de plus de $10 \mathrm{~cm}$ ) saines ou avortées (Deheuvels, 2011) ont été relevés. Les cabosses mûres ont été dénombrées et récoltées régulièrement pour avoir le nombre de cabosses produites par cacaoyer, le nombre de cabosses rongées ou pourries et le nombre de cabosses saines récoltées.

Pour étudier le lien entre la qualité des cabosses et la productivité, un échantillon de 24 cabosses (au plus) a été prélevé par individu. Dans cette zone, où la récolte s'effectue de septembre à décembre (exceptionnellement janvier), des cabosses ont été prélevées pendant ces 4 mois (au plus 6 cabosses mûres par mois). Leur masse et leur forme ont été déterminées, puis la masse et le nombre de fèves par cabosse, la forme des fèves, le rapport entre le diamètre de la cavité interne et le diamètre de la cavité externe du fruit.

La forme des cabosses $(F C a)$ a été déterminée par:

$$
F C a=l / L,
$$

avec $l$ la largeur externe de la cabosse et $L$ sa longueur externe (Fig. 4). La cabosse a une forme allongée lorsque $F C a$ tend vers 0 et une forme sphérique quand $F C a$ tend vers 1 (Zoro Bi et al., 2003).

Après écabossage, le rapport (Rcav en \%) entre le diamètre de la cavité externe et le diamètre de la cavité interne au niveau de la section la plus large a été déterminé :

$$
\operatorname{Rcav}=\frac{h}{l} \times 100
$$

avec $h$ la largeur de la cabosse en considérant les bords internes (diamètre interne) et $l$ la largeur de la cabosse en considérant les bords externes (diamètre externe) (Fig. 5). Ce rapport permet de renseigner la proportion de la loge des fèves dans la cabosse (Zoro Bi et al., 2003). 
Tableau 2. Liste des espèces recensées dans les trois SAFc.

Table 2. List of species recorded in the three AFSc.

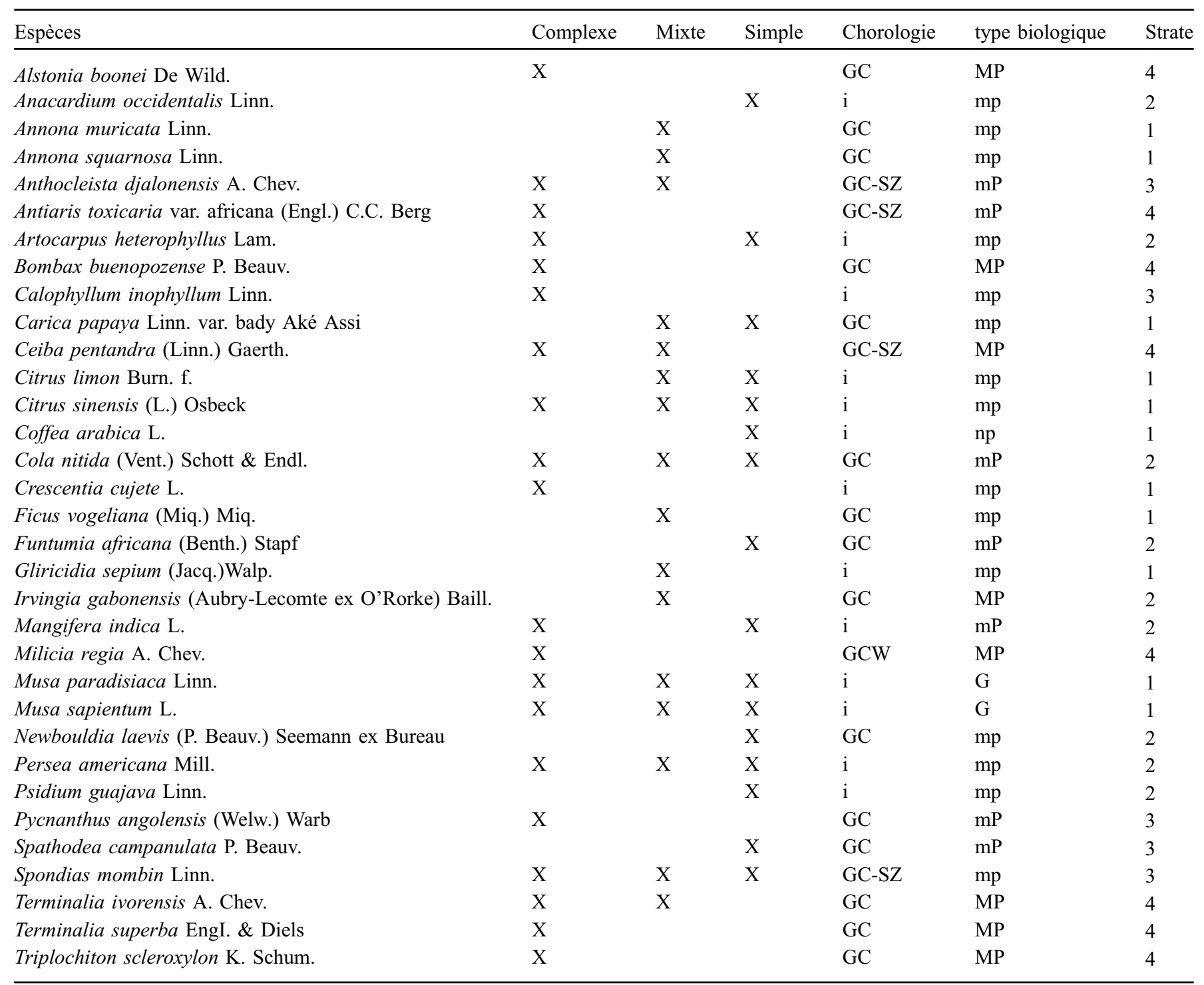

Liste incluant la chorologie (GC: espèce qui se rencontre dans la région guinéo-congolaise ; GCW : espèce endémique à l'Afrique de l'Ouest ; GC-SZ : espèce de la région guinéo-congolaise et soudano-zambézienne), le type morphologique (G : géophyte ; np : nanophanérophyte [hauteur de 0,25 à $2 \mathrm{~m}$ ]; $\mathrm{mp}:$ microphanérophyte [2 à $8 \mathrm{~m}$ ]; $\mathrm{mP}:$ mésophanérophyte [8 à $32 \mathrm{~m}$ ]; MP : mégaphanérophyte [plus de $32 \mathrm{~m}$ ]); Strate $1:$ basse $(2$ à $4 \mathrm{~m})$; Strate 2 : moyenne $(4$ à $8 \mathrm{~m})$; Strate $3:$ supérieure $(8$ à $16 \mathrm{~m})$; Strate 4 : émergente (plus de $16 \mathrm{~m})$.

Le nombre total de fèves par cabosse (fèves saines + fèves plates) a été compté et leur masse fraîche déterminée. Leur masse sèche a été calculée avec un coefficient de transformation à la masse fraîche de 0,34 (Essola, 2014).

Le nombre de fèves mal formées a été noté et 50 fèves ( 10 par cacaoyer) ont été sélectionnées aléatoirement. On a déterminé la forme de ces fèves (longueur, largeur et épaisseur). L'estimation de la production par parcelle a été faite à partir de la densité des cacaoyers, du nombre de cabosses produites par cacaoyer et de la masse des fèves sèches par cabosse.

\subsection{Analyses statistiques}

Après des analyses descriptives, pour apprécier les éventuelles variations entre les cacaoyers sous ombrage et sans ombrage, et entre variétés, des tests de comparaison de moyennes ont été utilisés. Le test $t$ de Student a été appliqué pour les données présentant une distribution normale et une homogénéité des variances. Dans le cas contraire, nous avons appliqué le test de Mann-Whitney. Des tests d'analyse de variance (ANOVA) ont été appliqués aux paramètres agronomiques (coussinets, cabosses et fèves); le test de comparaison multiple de Tukey a été utilisé pour identifier les différences. 


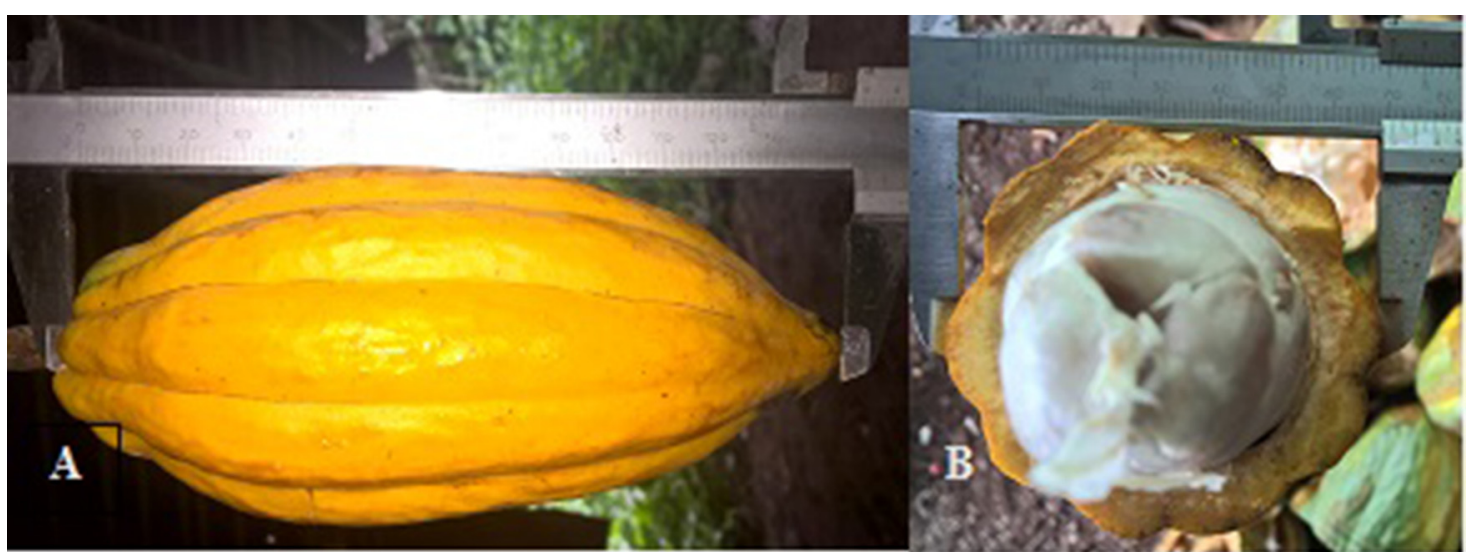

Fig. 4. Mesure de la longueur (A) et de la largeur (B) d'une cabosse.

Fig. 4. Measure of the length (A) and width (B) of a pod.

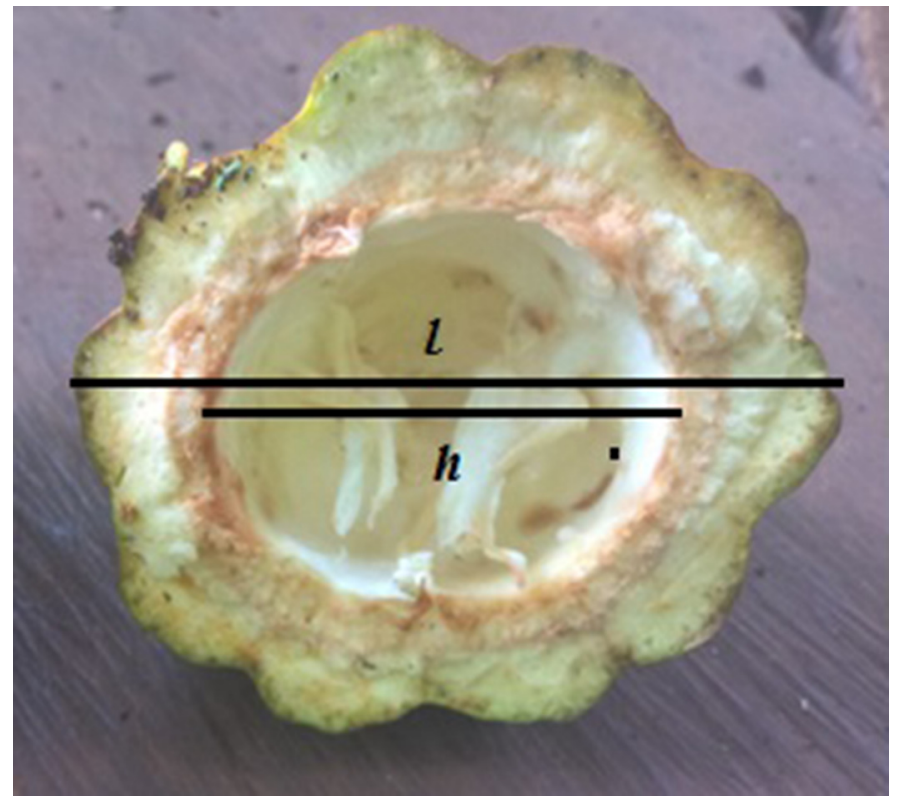

Fig. 5. Détermination du pourcentage de cavité.

Fig. 5. Determination of the cavity percentage.

Pour les données ne présentant pas de normalité ou d'homogénéité, le test de Kruskal-Wallis a été utilisé; lorsqu'il existait une différence significative, le test de comparaison multiple de Dunn a été utilisé. En outre, des tests de corrélation ont été effectués. Le niveau de significativité $p=0,05$ a été retenu. Les logiciels Xlstat 2014 et R 3.4.3 ont été utilisés.

\section{Résultats}

\subsection{Caractères phénotypiques liés à la floraison}

Nous avons compté en moyenne $71,9 \pm 10,7$ coussinets floraux par cacaoyer à une hauteur inférieure à $2 \mathrm{~m}$. Ce nombre varie significativement, de 81 pour les cacaoyers sans ombrage à 55 pour les cacaoyers sous ombrage, et en fonction de la variété cultivée : 82 coussinets pour « cacao Ghana » contre 37 pour «cacao français». Il varie également significativement, de 36 dans les SAFc mixtes à 107 dans les SAFc simples.

\subsection{Caractères phénotypiques liés aux fruits}

Dans l'ensemble des plantations visitées, le nombre de chérelles produites est en moyenne de 31,3 $\pm 4,9$ et ne varie statistiquement pas quel que soit le système considéré. Cependant, le nombre moyen de chérelles viables (ayant plus de $10 \mathrm{~cm}$ de long) est de $14 \pm 2,5$. Il est plus important dans les $\mathrm{SAFc}$ simples (Tab.3). Il est également influencé par l'ombrage, avec une valeur moyenne de 16 chérelles pour les cacaoyers sans ombrage contre 10 pour les cacaoyers sous ombrage.

Les cacaoyers étudiés produisent chacun en moyenne $9,5 \pm 1,8$ cabosses mûres par an. Ce nombre varie significativement d'un système à l'autre. Les plus grandes quantités sont enregistrées dans les SAFc mixtes et simples. La production des parcelles est estimée à 9314 cabosses/ha en moyenne. Cette quantité de cabosses récoltées varie significativement d'un SAFc à l'autre (Tab.3). 
Tableau 3. Paramètres agronomiques des cacaoyers dans les trois SAFc.

Table 3. Agronomic parameters of cocoa trees in the three AFSc.

\begin{tabular}{|c|c|c|c|c|c|c|}
\hline Système agroforestier & & Coussinets & Chérelles & Chérelles viables & Cabosses mûres/cacaoyer & Nombre de cabosses/ha \\
\hline Mixte & & $35,8^{\mathrm{a}}$ & $30,4^{\mathrm{a}}$ & $16,4^{\mathrm{a}, \mathrm{b}}$ & $13,3^{b}$ & $11369^{b}$ \\
\hline & $F$ & 16,6 & 2,0 & 5,5 & 5,01 & 5,88 \\
\hline ANOVA & $p$-value & $<0,0001$ & 0,1 & 0,005 & 0,007 & 0,003 \\
\hline
\end{tabular}

Dans une même colonne, les valeurs portant la même lettre sont statistiquement identiques.

Tableau 4. Caractéristiques des cabosses dans les trois SAFc. Table 4. Characteristics of pods in the three AFSc.

\begin{tabular}{lccc}
\hline $\begin{array}{l}\text { Système } \\
\text { agroforestier }\end{array}$ & $1 / \mathrm{L}$ & $\mathrm{h} / 1$ & $\begin{array}{l}\text { Masse moyenne } \\
\text { d'une cabosse }(\mathrm{g})\end{array}$ \\
\hline Simple & $0,52^{\mathrm{a}}$ & $65,28^{\mathrm{a}}$ & $542^{\mathrm{c}}$ \\
Mixte & $0,57^{\mathrm{b}}$ & $66,72^{\mathrm{b}}$ & $436^{\mathrm{a}}$ \\
Complexe & $0,56^{\mathrm{b}}$ & $66,41^{\mathrm{a}, \mathrm{b}}$ & $476^{\mathrm{b}}$ \\
$\mathrm{F}$ & $22,001^{\mathrm{b}}$ & 4,953 & 20,931 \\
$\operatorname{Pr}(>\mathrm{F})$ & $<0,0001$ & $0,007^{2}$ & $<0,0001$ \\
\hline
\end{tabular}

$1 / \mathrm{L}$ : rapport de la largeur sur la longueur de la cabosse $; \mathrm{h} / \mathrm{L}$ : rapport entre le diamètre interne et le diamètre externe de la cabosse, sur la section de la cabosse en son endroit le plus large.

Dans une même colonne, les valeurs portant la même lettre sont statistiquement identiques.

La longueur externe, la largeur externe et le diamètre de la cavité interne des cabosses sont en moyenne respectivement de $156 \mathrm{~mm}, 84 \mathrm{~mm}$ et de $55 \mathrm{~mm}$. Ces valeurs varient significativement en fonction de la variété cultivée. Elles sont respectivement de $163 \mathrm{~mm}, 85 \mathrm{~mm}$ et $56 \mathrm{~mm}$ pour la variété «cacao Ghana», contre $140 \mathrm{~mm}, 81 \mathrm{~mm}$ et $54 \mathrm{~mm}$ pour la variété « cacao français».

Le rapport entre la largeur et la longueur des cabosses est en moyenne de 0,5 $\pm 0,01$ et varie significativement de 0,52 dans les SAFc simples à 0,57 dans les SAFc mixtes.

Le pourcentage de cavité des cabosses varie entre 50,6 et $79,2 \%$. La valeur moyenne par cabosse est de $66,1 \pm 5,0 \%$ et varie significativement de $65,3 \%$ dans les SAFc simples à $66,7 \%$ dans les SAFc mixtes.

La masse moyenne d'une cabosse est de $487 \pm 4 \mathrm{~g}$ et varie de $111 \mathrm{~g}$ à $1236 \mathrm{~g}$ sur l'ensemble des cabosses récoltées. Elle varie significativement, de $518 \mathrm{~g}$ pour les cabosses de variété « cacao Ghana » à 397 g pour la variété « cacao français ». Elle varie significativement, de $436 \mathrm{~g}$ dans les SAFc mixtes à $541 \mathrm{~g}$ dans les SAFc simples (Tab. 4).

\subsection{Caractères phénotypiques liées aux fèves}

Le rapport largeur/longueur des fèves est en moyenne de $0,52 \pm 0,04$. Ce rapport ne varie pas significativement selon les SAFc considérés. L'épaisseur des fèves a une valeur moyenne de $5,4 \mathrm{~mm} \pm 0,08$. Elle varie de $2 \mathrm{~mm}$ pour les fèves les plus plates à $11 \mathrm{~mm}$ pour les plus ovales. La longueur, la largeur et l'épaisseur des fèves sont en moyenne respectivement de $21,66 \mathrm{~mm}, 11,17 \mathrm{~mm}$ et $5,35 \mathrm{~mm}$. Ces valeurs varient significativement en fonction de la variété cultivée. Elles sont respectivement de $22 \mathrm{~mm}, 11,31 \mathrm{~mm}$ et $5,45 \mathrm{~mm}$ pour la variété « cacao Ghana», contre $21 \mathrm{~mm}, 10,83 \mathrm{~mm}$ et $5,09 \mathrm{~mm}$ pour la variété «cacao français»

Le nombre de fèves par cabosse varie de 11 à 83 . Le nombre moyen de fèves par cabosse est de 39,6 $\pm 9,9$. Il ne varie pas significativement selon le système considéré (Tab. 5), ni d'une variété à l'autre.

Les fèves d'une cabosse ont une masse fraîche moyenne de $117,3 \pm 3,0 \mathrm{~g}$ et une masse sèche moyenne estimée à $39,9 \pm 1,0 \mathrm{~g}$. La masse moyenne de fèves fraîches d'une cabosse varie significativement, de 107,5 g dans les SAFc mixtes à $127,4 \mathrm{~g}$ dans les SAFc simples (Tab. 5). Elle varie significativement d'une variété à l'autre, de $122 \mathrm{~g}$ pour la variété «cacao Ghana» à $104 \mathrm{~g}$ pour la variété «cacao français ». Les cabosses échantillonnées comptent en moyenne $1,27 \pm 0,28$ fèves plates chacune. Ce nombre de fèves mal formées est le même dans tous les SAFc (Tab. 5), mais varie significativement selon la variété.

Le rapport de la masse des fèves sèches sur la masse de la cabosse (indice de récolte) varie d'un SAFc à l'autre. Il est le même $(0,088)$ dans les SAFc mixte et complexe, et de 0,082 dans les SAFc simples. Il varie également d'une variété à l'autre, de 0,083 pour la variété « cacao Ghana » à 0,092 pour la variété « cacao français».

Ces données ont servi à l'estimation de la production des différents SAFc. La masse de fèves sèches récoltées varie significativement d'un système à l'autre: $476 \mathrm{~kg} / \mathrm{ha}$ dans les SAFc simples contre $205 \mathrm{~kg} / \mathrm{ha}$ dans les SAFc complexes.

\section{Discussion}

\subsection{Importance des systèmes agroforestiers à base de cacaoyers}

Les systèmes agroforestiers traditionnels permettent aux paysans de s'approvisionner en ressources ligneuses et non ligneuses (Clark et Sunderland, 2004), pour couvrir leurs besoins en bois d'œuvre, en bois de chauffe, en aliments et en remèdes issus de plantes médicinales (Herzog, 1994; Sonwa et al., 2001 ; Adou Yao et al., 2016). Outre ces services directs, les SAFc participent à la conservation des sols, des eaux, du climat et de la biodiversité (Rice et Greenberg, 2000; Leakey, 2001 ; Clough et al., 2011). Les agro-forêts servent également de refuge pour des espèces animales et végétales (Schroth 
Tableau 5. Caractéristiques des fèves dans les trois SAFc.

Table 5. Characteristics of beans in the three AFSc.

\begin{tabular}{lllcl}
\hline $\begin{array}{l}\text { Système } \\
\text { agroforestier }\end{array}$ & $\begin{array}{l}\text { Nombre de } \\
\text { fèves/cabosse }\end{array}$ & $\begin{array}{l}\text { Forme des } \\
\text { fèves (1/L) }\end{array}$ & $\begin{array}{l}\text { Masse de fèves } \\
\text { fraîches/cabosse (g) }\end{array}$ & $\begin{array}{l}\text { Nombre de } \\
\text { fèves mal } \\
\text { formées/cabosse }\end{array}$ \\
\hline Simple & $40,6^{\mathrm{a}}$ & $0,51^{\mathrm{a}}$ & $127^{\mathrm{c}}$ & $\begin{array}{l}\text { Masse de fèves } \\
\text { sèches/ha (kg) }\end{array}$ \\
Mixte & $38,7^{\mathrm{a}}$ & $0,52^{\mathrm{a}}$ & $107^{\mathrm{a}} 7^{\mathrm{a}}$ & $1,23^{\mathrm{a}}$ \\
Complexe & $39,5^{\mathrm{a}}$ & $0,52^{\mathrm{a}}$ & $116^{\mathrm{b}}$ & $1,18^{\mathrm{a}}$ \\
F & 2,292 & 1,969 & 15,840 & 0,394 \\
Pr $(>\mathrm{F})$ & 0,102 & 0,140 & $<0,0001$ & 0,674 \\
\hline
\end{tabular}

Dans une même colonne, les valeurs portant la même lettre sont statistiquement identiques.

et al., 2004). Selon Ofori-Frimpong et al. (2007), au Ghana, les espèces de mammifères et d'oiseaux recensées dans des systèmes sous ombrage dense étaient trois fois plus nombreuses que dans des systèmes de plein soleil. Par ailleurs, les SAFc les plus complexes séquestrent plus de carbone (Nair, 2012), contribuant ainsi à atténuer le réchauffement climatique (Gockowski et Sonwa, 2011; Somarriba et al., 2013). Ces systèmes agroforestiers traditionnels diffèrent de ceux proposés par les organismes de certification du cacao ivoirien et de recherche telles que le CNRA (Centre national de recherche agronomique) (Sanial, 2014; Smith-Dumont et al., 2014), lesquels se résument à associer un nombre limité d'espèces sélectionnées. Plus un système est complexe, plus il rend divers services écosystémiques dont il faut tenir compte, en plus de la production du cacao. Cependant, cette complexité tend à limiter et à réduire la productivité des cacaoyers.

\subsection{Productivité du cacaoyer et type de système agroforestier}

Les différents systèmes étudiés utilisent des semences de cacaoyer d'origines diverses, un mélange de variétés améliorées et non améliorées (Kpangui et al., 2015). Cette utilisation de cacaoyers d'origines diverses contribue, en général, à une faible production de ces systèmes (Assiri et al., 2009). L'abondante floraison observée dans les SAFc simples comparativement aux autres systèmes est due à la nature du matériel végétal utilisé par les paysans. En effet, les résultats obtenus montrent que les variétés « cacao Ghana» produisent beaucoup plus de coussinets floraux que les variétés «cacao français ». En outre, la présence d'ombrage dans les SAFc complexes et mixtes réduit et retarde la floraison du cacaoyer (Burle, 1961). Mais malgré une forte production de coussinets floraux observée dans les SAFc simples par rapport aux autres systèmes, une même quantité de chérelles est produite dans les différents SAFc. En effet, sur le nombre total de fleurs produites sur un arbre, peu de fruits arrivent au stade de chérelles (Boyer, 1974). Cela est dû, selon Deheuvels (2011), au fait que la pollinisation n'affecte que $2 \%$ des fleurs. À ce fait s'ajoute la corrélation non significative observée entre le nombre de coussinets formés et le nombre de cabosses formées $(\mathrm{r}=0,26)$, qui révèle qu'une forte floraison n'implique pas obligatoirement une forte production de fruits. Les travaux de Lachenaud (1991), réalisés à Divo sur des cacaoyers de variétés diverses, ont également montré que l'intensité de la floraison n'était pas significativement corrélée avec le nombre de fruits du cacaoyer $(\mathrm{r}=-0,17)$.

Un plus grand nombre de chérelles viables est observé dans les SAFc simples comparativement aux autres systèmes. Logiquement, du fait de la plus forte production de chérelles, on devrait aboutir au constat d'un nombre plus élevé de cabosses mûres dans les $\mathrm{SAFc}$ simples. Les résultats montrent cependant que les SAFc mixtes et simples produisent le même nombre de cabosses mûres à moins de $2 \mathrm{~m}$ par cacaoyer. Cela est attribué au phénomène de «flétrissement» ou wilt en anglais ou encore «wilt physiologique» (Deheuvels, 2011; Jagoret, 2011). Ce taux de flétrissement des fruits n'est pas corrélé avec la quantité de cabosses produites (Lachenaud, 1991). Il est considéré comme un mécanisme physiologique de régulation de la production (Braudeau, 1969; Paulin, 1981 ; Wood et Lass, 1985; Lachenaud, 1991). En effet, une grande proportion de fruits ne parvient pas à maturité, mais flétrit et se dessèche sur l'arbre (Boyer, 1974). Selon Lachenaud (1991), ce phénomène physiologique ne concerne que les jeunes fruits jusqu'à environ 70 jours, parfois exceptionnellement jusqu'à 100 jours, et peut affecter 20 à $90 \%$ des chérelles.

Les résultats de la présente étude s'opposent à ceux de Hutcheon (1976; 1981), Soria (1977), Jadin et Paulin (1987). Pour Hutcheon $(1976 ; 1981)$, la production des fruits est contrôlée par l'intensité de la floraison, le flétrissement des fruits et la croissance des cabosses. Quant aux travaux de Soria (1977), ils ont mis en évidence une corrélation très forte $(r=0,916)$ entre la floraison et la fructification. Jadin et Paulin (1987) ont conclu que l'augmentation de floraison est responsable des augmentations de rendement. Ces auteurs ont cependant abouti à ces résultats avec un matériel végétal homogène.

Le taux moyen de chérelles ayant effectivement atteint la maturité et récoltées est plus grand dans les SAFc simple et mixte que dans les SAFc complexes. Ce faible taux dans les SAFc complexes peut être attribué à l'origine des semences. En effet, les cacaoyers dans les SAFc complexes, en général de variété «cacao français», sont moins productifs que les cacaoyers dits «cacao Ghana» (semences génétiquement améliorées et/ou hybridées) utilisés dans les systèmes mixtes et simples. Par ailleurs, la complexité de ces SAFc favorise la présence de rongeurs nuisibles (écureuils, rats) (Kouakou, 2015). Ces rongeurs s'attaquent aux cabosses mûres ou presque mûres, les perforent, font tomber leurs fèves au sol et se nourrissent du mucilage (Bellier et Lefèvre, 1968), 
diminuant encore la productivité des cacaoyers dans les $\mathrm{SAFc}$ complexes.

Le faible nombre de cabosses récoltées dans les SAFc complexes est également lié aux conditions environnementales (pluviométrie, humidité, densité d'arbres associés, etc.), qui influencent fortement la productivité d'un cacaoyer. Cette productivité peut être pratiquement nulle en cas d'ombrage trop important (Burle, 1961). Des études ont montré que l'ombrage réduit les rendements (Braudeau, 1969) et ne serait pas nécessaire pour une culture intensive (Lachenaud, 1991). En effet, l'ombrage modifie la quantité de lumière, les températures et les mouvements d'air dans la cacaoyère et affecte directement la photosynthèse, la croissance et le rendement du cacaoyer (de Zuidema et al., 2005 ; de Almeida et Valle, 2007). En outre, Boulay (1998) affirme qu'un ombrage excessif crée un microclimat plus humide, ce qui favorise la prolifération de maladies telles que la pourriture brune, et réduit la production (Mossu, 1990).

L'une des raisons qui militent en faveur de l'ombrage est que la longévité des cacaoyers est moindre dans les systèmes plein soleil que sous ombrage (Ahenkorah et al., 1974). En outre, la zone de contact forêt-savane où a eu lieu cette étude présente une faible, voire une médiocre, fertilité des sols (Avenard et al., 1973) et des conditions climatiques avec une saison sèche de plus de 2 mois. Tout cela nécessite, selon Boulay (1998), l'augmentation de la densité des arbres d'ombrage dans les SAFc. Les SAFc mixtes apparaissent ici, en termes de production, comme un bon compromis entre $\mathrm{SAFc}$ simples et complexes. En effet, la présence modérée d'arbres associés dans ces systèmes réduit les risques d'ombrage excessif et les effets de concurrence pour les ressources nutritives du sol. Au-delà de cet aspect, certaines de ces espèces telles que le fromager (Ceiba pentandra), le fraké (Terminalia superba) et Newbouldia laevis sont associées par les paysans pour leur effet fertilisant ou leur action humidifiante sur le microclimat en saison sèche (Tayo, 2014 ; Adou Yao et al., 2016). Cependant, certaines structures, comme l'ANADER (Agence nationale d'appui au développement rural) et le CNRA, déconseillent certains de ces arbres jugés bénéfiques par les paysans (Sanial, 2014). On manque de preuves scientifiques sur l'intérêt de ces arbres (Smith-Dumont et al., 2014).

Le type de système n'influe pas significativement sur les dimensions des cabosses ni sur celles des fèves. Par contre, ces paramètres dépendent de la variété du cacaoyer. Les cabosses produites par les cacaoyers de variété «cacao Ghana» sont plus volumineuses que les cabosses de variété «cacao français » et produisent des fèves de masse supérieure. Les paysans font une distinction entre ce qu'ils appellent l'« ancien Ghana», supportant l'ombrage, et le «nouveau Ghana», exigeant une culture sans ombrage (Kpangui et al., 2015). La variété «ancien Ghana» remplace progressivement la variété « cacao français» (Amelonado) dans les systèmes complexes. Ce fait traduirait un abandon progressif des SAFc complexes (Ruf, 2011; Kpangui et al., 2015) au profit de systèmes utilisant du matériel génétiquement amélioré à haut rendement et ne nécessitant pas d'ombrage.

S'agissant des fèves, le type de SAFc n'influe ni sur leur forme, ni sur leur nombre, ni sur le nombre de fèves plates dans les cabosses. L'ombrage n'influe donc pas sur ces paramètres. Les fèves plates observées sont des graines ayant avorté tardivement, ou contenant des embryons immatures (Lachenaud, 1991). Concernant le nombre de fèves par cabosse, nos résultats montrent une fluctuation du nombre de fèves entre 11 et 83 et une valeur moyenne de fèves de 40 . Cette grande variabilité du taux de remplissage des cabosses est imputable selon Braudeau (1969) au taux de fécondation, aux conditions de nutrition (particulièrement en oligo-éléments) et aux caractéristiques variétales de la plante.

\section{Conclusion}

La présente étude a permis de mettre en évidence l'influence de la variété, de l'ombrage et du type de système agroforestier sur les caractères phénotypiques et la productivité du cacaoyer, notamment ceux liés à la formation des cabosses et de leurs fèves. Elle montre que la quantité, la masse et la qualité des cabosses sont significativement plus grandes dans les systèmes à plus faible ombrage et qui associent très peu d'autres espèces aux cacaoyers. Par contre, le nombre de fèves par cabosse ne varie pas significativement quel que soit le système. Par ailleurs, elle met en lumière la faible productivité des cacaoyers dans les SAFc complexes. Celle-ci est liée aussi bien à la variété utilisée dans ces systèmes complexes qu'à l'influence de l'ombrage. L'association de ces deux facteurs conduit les paysans à s'orienter de plus en plus vers les variétés hybrides à fort rendement et pouvant se cultiver sous ombrage modéré ou en plein soleil.

La seule intention d'accroître la production de cacao conduirait donc à l'adoption des $\mathrm{SAFc}$ simples et à l'abandon des $\mathrm{SAFc}$ complexes et de leur riche diversité végétale, et donc des services qu'ils procurent aux paysans ainsi qu'à la communauté. Toutefois, le SAFc simple pose des questions environnementales, telles que la faible valeur et la faible densité d'espèces associées indigènes et/ou exotiques. Il serait donc difficile, dans cette zone où l'association avec l'arbre existe depuis plusieurs générations, de proposer ce type de système comme modèle de culture durable du cacaoyer. Audelà de la production des cacaoyers, la présence de l'arbre d'ombrage associé est en effet important à considérer pour les services qu'elle rend aux paysans et à la communauté.

Remerciements. Les auteurs réitèrent leurs remerciements aux populations villageoises, notamment aux paysans qui ont bien voulu mettre leurs plantations à disposition. Ces remerciements s'adressent également au Programme d'appui stratégique pour la recherche scientifique (PASRES) et au Conseil ouest et centre africain pour la recherche et le développement agricole (CORAF) qui ont financé la réalisation des études.

\section{Références}

Adou Yao CY, Kpangui KB, Vroh BTA, Ouattara D. 2016. Pratiques culturales, valeurs d'usage et perception des paysans des espèces compagnes du cacaoyer dans des agroforêts traditionnelles au centre de la Côte d'Ivoire. Revue d'Ethnoécologie 9. Disponible sur http://ethnoecologie.revues.org/2474.

Ahenkorah Y, Akrofi GS, Adri AK. 1974. The end of the first cacao shade and manurial experiment at the Cacao Research Institute of Ghana. J Hort Sci 49: 43-51.

Assiri AA. 2010. Étude de la régénération cacaoyère en côte d'ivoire : impact des techniques de réhabilitation et de replantation sur le 
développement et la productivité des vergers de cacaoyers (Theobroma cacao L.) en relation avec l'état du sol. Thèse de Doctorat. Université de Cocody, Abidjan, 170 p.

Assiri AA, Yoro GR, Deheuvels O, Kébé BI, Kéli ZJ, Adiko A, et al. 2009. Les caractéristiques agronomiques des vergers de cacaoyer (Theobroma cacao L.) en Côte d'Ivoire. Journal of Animal \& Plant Sciences 2(1): 55-66.

Avenard J-M, Bonvallot J, Latham M, Renard-Dugerdil M, Richard J. 1973. Le contact forêt-savane en moyenne Côte-d'Ivoire. Annales de Géographie 82(453): 513-544.

Bardin A. 1937. Le cacaoyer en Côte d'Ivoire. Annales Agricoles de l'Afrique Occidentale Française et Etrangère 1(2): 135-150.

Bellier L, Lefèvre C. 1968. Les rongeurs nuisibles au cacaoyer. Rapport préliminaire. Paris (France): ORSTOM-IFCC, 15 p.

Blanc-Pamard C. 1978. Espace vécu et milieu de contact forêt-savane chez les paysans Baoulé et leurs enfants dans le Sud du «V Baoulé», Côte d'Ivoire. Cahiers ORSTOM, Série Sciences Humaines 15(2): 145-172.

Boulay M. 1998. Étude de la phénologie de différents hybrides de cacaoyer associés à six espèces d'arbres d'ombrage, Mémoire de maître ès sciences. Université Laval, Canada, $74 \mathrm{p}$.

Boyer J. 1974. Étude écophysiologique du développement de cacaoyers cultivés au Cameroun. Café Cacao Thé 18(1): 30 p.

Braudeau J. 1969. Le cacaoyer. Collection Techniques agricoles et productions tropicales. Paris (France) : Maisonneuve et Larose, $304 \mathrm{p}$.

Burle L. 1961. Le cacaoyer. (Tome premier). Paris (France): Maisonneuve et Larose, $316 \mathrm{p}$.

Camara A, Dugué P, Cheylan JP, Kalms JM. 2009. De la forêt naturelle aux agroforêts en Guinée forestière. Cahiers Agricultures 18(5): 425-432.

Chauveau JP. 1979. Les cadres socio-historiques de la production dans la région de Kokumbo (pays baoulé, Côte d'Ivoire). Cahiers ORSTOM, Série Sciences humaines 7: 143.

Cissé A. 2013. Caractérisation phytoécologique et estimation de la biomasse des espèces arborescentes des agroforêts à base de cacao du département de Lakota (Centre-Ouest, Côte d'Ivoire). Mémoire de Diplôme d'études approfondies de botanique. Université Félix Houphouët-Boigny, Abidjan, 64 p.

Clark EL, Sunderland TC. 2004. The key non-timber Forest Products of central Africa: state of the Knowledge. USAID, Bureau for Africa, Office of Sustainable Development, Technical paper $\mathrm{n}^{\mathrm{o}} 122$, $186 \mathrm{p}$.

Clough Y, Barkmann J, Juhrbandt J, Kessler M, Wanger TC, Anshary A, et al. 2011. Combining high biodiversity with high yields in tropical agroforests. Proceedings of the National Academy of Sciences 108(20): 8311-8316.

de Almeida AAF, Valle RR. 2007. Ecophysiology of the cacao tree. Brazilian Journal of Plant Physiology 19(4): 425-448.

Deheuvels O. 2011. Compromis entre productivité et biodiversité sur un gradient d'intensité de gestion de systèmes agroforestiers à base de cacaoyers de Talamanca, Costa Rica, Thèse de Doctorat. SUPAGRO, Montpellier, France, 185 p.

Essola ELC. 2014. Évaluation des rendements potentiels en cacao (Theobroma cacao L.) dans les systèmes agroforestiers complexes en zone forestière à pluviométrie bimodale du centre Cameroun. Mémoire de diplôme d'ingénieur agronome. Université de Dschang, Ebolowa, Cameroun, 64 p.

Freud EH, Petithuguenin P, Richard J. 2000. Les champs de cacao : un défi de compétitivité Afrique-Asie. Paris: Karthala et CIRAD, $207 \mathrm{p}$.

Gockowski J, Sonwa D. 2011. Cocoa intensification scenarios and their predicted impact on $\mathrm{CO}_{2}$ emissions, biodiversity conserva- tion, and rural livelihoods in the Guinea rain forest of West Africa. Environmental Management 48: 307-321.

Guillaumet JL, Adjanohoun E. 1971. La végétation de la Cote d'Ivoire. In: Le milieu naturel de Côte d'Ivoire. Mémoires ORSTOM, 50. Paris (France) : Orstom, pp. 161-263.

Herzog F. 1994. Multipurpose shade trees in coffee and cocoa plantations in Côte d'Ivoire. Agroforestry Systems 27: 259-267.

Hutcheon WV. 1976. A framework for the physiology of cocoa (part 2). Cocoa Growers' Bulletin (Bournville) 25: 5-10.

Hutcheon WV. 1981. Physiological studies on cocoa (Theobroma cacao L.) in Ghana. PhD thesis. University of Aberdeen, 599 p.

Jadin P, Paulin D. 1987. Étude des facteurs de production liés à la biologie florale influencés par la fertilisation minérale et (ou) l'irrigation sur cacaoyers adultes. $10^{\mathrm{e}}$ Conférence internationale sur les recherches cacaoyères. Lagos : Cocoa Producers' Alliance, pp. 253-258.

Jagoret P. 2011. Analyse et évaluation de systèmes agroforestiers complexes sur le long terme : application aux systèmes de culture à base de cacaoyer au Centre Cameroun. Thèse de doctorat. SUPAGRO, Montpellier, France, 236 p.

Jagoret P, Bouambi E, Menimo T, Domkam I, Batomen F. 2008. Analyse de la diversité des systèmes de pratiques en cacaoculture : cas du Centre Cameroun. Biotechnologie, Agronomie, Société et Environnement 12(4): 367-377.

Kouakou CV. 2015. Diversité de la faune mammalienne dans les plantations agroforestières à cacao du Centre de la Côte d'Ivoire : cas de la sous-préfecture de Kokumbo. Mémoire de Master. Université Jean Lorougnon Guédé, Daloa, Côte d'Ivoire, 56 p.

Kpangui KB. 2015. Dynamique, diversité végétale et valeurs écologiques des agroforêts à base de cacaoyers de la souspréfecture de Kokumbo (centre de la Côte d'Ivoire). Thèse de Doctorat. Université Félix Houphouët-Boigny, Abidjan, 187 p.

Kpangui KB, Kouamé D, Gone Bi ZB, Vroh BTA, Koffi BJC, Adou Yao CY. 2015. Typology of cocoa-based agroforestry systems in a forest-savannah transition zone: case study of Kokumbo (Centre, Côte d'Ivoire). International Journal of Agronomy and Agricultural Research (IJAAR) 6(3): 36-47.

Lachenaud P. 1991. Facteurs de la fructification chez le cacaoyer (Theobroma cacao L.) : influence sur le nombre de graines par fruit. Thèse de doctorat de l'Institut national agronomique ParisGrignon, 186 p.

Leakey RB. 2001. Win-win land use strategies for Africa: 2, Capturing economic and environment benefits with multistrata agroforests. International Forestry Review 3: 331-340.

Mossu G. 1990. Le cacaoyer. Collection Le technicien d'agriculture tropicale. Paris : Maisonneuve et Larose, $160 \mathrm{p}$.

Nair PK. 2012. Carbon sequestration studies in agroforestry systems: a reality-check. Agroforestry Systems 86: 243-253.

Ofori-Frimpong K, Asase A, Yelibo MA. 2007. Cocoa farming and biodiversity in Ghana. Annual report. Earth Watch Institute, $34 \mathrm{p}$.

Paulin D. 1981. Contribution à l'étude de la biologie florale du cacaoyer. Bilan de pollinisations artificielles. Café Cacao Thé 25 (2): $105-112$.

Peltre P. 1977. Le «V Baoulé» (Côte d'Ivoire centrale): héritage géomorphologique et paléoclimatique dans le tracé du contact forêt-savane. Paris : Mémoires ORSTOM, 193 p.

Perraud A. 1971. Les sols. In : Le Milieu naturel de la Côte d'Ivoire. Paris : Mémoires ORSTOM, nº 50, pp 263-327.

Piba SC. 2008. Apport de la flore naturelle dans la vie de la population d'une région cacaoyère en Côte d'Ivoire: cas du département 
d'Oumé. Mémoire de Diplôme d'études approfondies de botanique, Université Félix Houphouët-Boigny, Abidjan, 64 p.

Rice R, Greenberg R. 2000. Cacao cultivation and the conservation of biological diversity. AMBIO: A Journal of the Human Environment 29: 167-173.

Ruf FO, Schroth G. 2004. Chocolate forests and monocultures: a historical review of cocoa growing and its conflicting role in tropical deforestation and forest Conservation. In: Agroforestry and biodiversity conservation in tropical landscapes. Washington DC (USA): Island Press, pp. 107-134.

Ruf FO. 2011. The myth of complex cocoa agroforests: the case of Ghana. Hum Ecol 39: 373-388.

Sanial E. 2014. À la recherche de l'ombre: analyse du retour des arbres associés dans les plantations de cacao ivoiriennes. Mémoire de master. Université Jean Moulin Lyon 3, 203 p.

Schroth G, da Fonseca GAB, Harvey CA, Gascon C, Vasconcelos HL, Izac A-MN. 2004. Agroforestry and biodiversity conservation in tropical landscapes. Washington DC (USA): Island Press, $537 \mathrm{p}$.

Smith-Dumont E, Gnahoua GM, Ohouo L, Sinclair FL, Vaast P. 2014. Farmers in Côte d'Ivoire value integrating tree diversity in cocoa for the provision of ecosystem services. Agroforestry System 88(6): 1047-1066.

Somarriba E, Cerda R, Orozco L, Cifuentes M, Espin T, Mavisoy H, et al. 2013. Carbon stocks and cocoa yields in agroforestry systems of Central America. Agriculture, Ecosystems and Environment 173: 46-57.

Sonwa DJ, Weise SF, Tchatat M, Nkongmeneck BA, Adesina AA, Ndoye $\mathrm{O}$, et al. 2001. Rôle des agroforêts à cacao dans la foresterie paysanne et communautaire au sud-Cameroun. Document RDFN, $\mathrm{n}^{\mathrm{o}} 25 \mathrm{~g}(\mathrm{i}), 12 \mathrm{p}$.

Soria S de J. 1977. Dinâmica populacional de Forcipomyia spp (Diptera, Ceratopogonidae) na Bahia, Brasil. II: Variaveis bióticas relacionadas com a polinização do cacaueiro. Revista theobroma (Ilhéus) 7: 19-33.

Tayo GKY. 2014. Dynamique de la biodiversité ligneuse et des stocks de carbone dans les systèmes agroforestiers à base de cacaoyer au centre Cameroun: cas de Ngomedzap. Mémoire de master. Université de Dschang, Cameroun, 91 p.

Wood GAR, Lass RA. 1985. Cocoa (4th edition). Tropical agriculture series. London: Longman, 620 p.

Zoro Bi I, Koffi KK, Djè Y. 2003. Caractérisation botanique et agronomique de trois espèces de cucurbites consommées en sauce en Afrique de l'Ouest: Citrullus sp., Cucumeropsis mannii Naudin et Lagenaria siceraria (Molina) Standl. Biotechnologie Agronomie, Société et Environnement 7: 189-199.

Zuidema PA, Leffelaar AF, Gerritsma W, Mommer K, Anten NPR. 2005. A physiological production model for cocoa (Theobroma cacao): model presentation, validation and application. Agricultural System 84: 195-225.

Citation de l'article : Kouadio V-PG, Vroh BTA, Kpangui KB, Kossonou ASF, Adou Yao CY. 2018. Incidence de 1'ombrage sur les caractères phénotypiques du cacaoyer en zone de transition forêt-savane au centre de la Côte d'Ivoire. Cah. Agric. $27: 55001$. 